\title{
Mecanismos para Suporte à Auto-Regulação da Aprendizagem do Estudante
}

\author{
Allen Fernando Oberleitner Lima ${ }^{1}$, Edson P. Pimentel ${ }^{1}$ \\ ${ }^{1}$ Universidade Federal do ABC (UFABC) \\ Santo André - SP - Brasil \\ allenfol@yahoo.com.br, edson.pimenteldufabc.edu.br
}

\begin{abstract}
Resumo - A arquitetura pedagógica da sala de aula tradicional, transposta para a aprendizagem eletrônica - e-learning, segue um fluxo em que o professor entrega materiais instrucionais aos estudantes e periodicamente realiza avaliações, com pouco envolvimento reflexivo do estudante no decorrer do processo. A busca por estratégias que envolvam ativamente o estudante passa pela incorporação de mecanismos de autoregulação conectados a um modelo de avaliação e acompanhamento da aprendizagem. Esses mecanismos não estão presentes em Ambientes Virtuais de Aprendizagem (AVAs) bastante utilizados no Brasil, como o Moodle e o Tidia-AE (Sakai). Este trabalho propõe a incorporação de mecanismos para auto-regulação da aprendizagem $e$ apresenta o seu funcionamento através da ferramenta SRS - Self Regulation System.
\end{abstract}

Palavras-chave: Auto-regulação, Aprendizagem, Avaliação, AVA, Metacognição.

Abstract - The architecture of teaching traditional classroom, implemented into the elearning, following a stream where the teacher delivers instructional materials to students and periodically conducts evaluations, with little involvement reflective of the student throughout the process. The search for strategies that actively involve the student goes through the incorporation of self-regulatory mechanisms connected a model of evaluation and monitoring of learning. These mechanisms are not present in Virtual Learning Enviroments (VLEs) widely used in Brazil, such as Moodle and Tidia$A E$ (Sakai).This work proposes the incorporation of mechanisms for self-regulated learning and in its operation through the tool SRS-Self Regulation System.

Keywords: Self-regulation, Learning, Assessment, LMS, Metacognition

\section{Introdução}

A arquitetura pedagógica da sala de aula tradicional segue um fluxo em que o professor organiza materiais instrucionais, entrega aos estudantes e periodicamente realiza avaliações do processo. A transposição para o e-learning - aprendizagem mediada por tecnologia da informação e comunicação - TDICs, não segue um fluxo muito diferente. A busca por estratégias que envolvam ativamente o estudante passa pela incorporação de mecanismos de auto-regulação no processo de ensino-aprendizagem. Esses mecanismos estariam diretamente relacionados, segundo Pimentel (2006), a um modelo de avaliação e acompanhamento da aprendizagem.

Os métodos de avaliação ocupam espaço relevante no conjunto das práticas pedagógicas aplicadas ao processo de ensino e aprendizagem. Avaliar, neste contexto, 
não se resume à mecânica do conceito formal e estatístico e nem simplesmente em atribuir notas, atividades obrigatórias à decisão de avanço ou retenção em determinadas disciplinas. É necessário um acompanhamento durante o processo [Zaiane e Luo, 2001]. Outro aspecto importante é fazer uso de mecanismos que ajudem o próprio estudante a se conscientizar e a identificar seus pontos falhos na aprendizagem. Pesquisas em Educação indicam que a aprendizagem efetiva acontece quando os aprendizes conseguem controlar a própria aprendizagem e se comprometem com o processo. $\mathrm{O}$ conhecimento sobre o próprio conhecimento é possível através do monitoramento da aprendizagem que é um dos aspectos da Metacognição, que em linhas gerais, constitui a habilidade das pessoas em monitorar, avaliar, planejar ou controlar a própria aprendizagem [Flavell, 1979].

Segundo Bransford, se os estudantes não conseguem diferenciar com precisão o que eles sabem do que eles não sabem, dificilmente irão conseguir se comprometer em atividades metacognitivas avançadas e, consequentemente, atingirem a auto-regulação da sua aprendizagem [Bransford, 2003].

Apesar da crescente evolução das Tecnologias Digitais da Informação e Comunicação (TDICs) aplicadas à Educação, nota-se em Ambientes Virtuais de Aprendizagem (AVAs) bastante utilizados no Brasil e no mundo, como o Moodle ${ }^{1}$ e Tidia-Ae/Sakai ${ }^{2}$, a inexistência de mecanismos capazes de propiciar a Auto-Regulação da Aprendizagem. A Tabela 1 traz um comparativo das ferramentas avaliativas nos AVA Moodle e Tidia-Ae.

Tabela 1 - Comparativo dos AVAs Moodle e Tidia-Ae quanto às ferramentas avaliativas

\begin{tabular}{|l|c|c|}
\cline { 2 - 3 } \multicolumn{1}{c|}{} & \multicolumn{2}{c|}{ AVA } \\
\cline { 2 - 3 } \multicolumn{1}{c|}{} & Moodle & Tidia-Ae \\
\hline Ferramenta Estudada & Questionário & Exercícios \\
\hline Banco de Questões & SIM & SIM \\
\hline Associação de Conteúdos & NÃO & NÃO \\
\hline Fluxo Ordenado das Avaliações & NÃO & NÃO \\
\hline Avaliação Cognitiva & SIM & SIM \\
\hline Avaliação Metacognitiva & NÃO & NÃO \\
\hline Métricas (Quadro de Notas) & Notas & Notas \\
\hline
\end{tabular}

Este trabalho propõe a incorporação de mecanismos para auto-regulação da aprendizagem e apresenta o seu funcionamento através da ferramenta SRS - Self Regulation System. Além disso, mostra um estudo comparativo de um cenário com e sem o uso dos mecanismos propostos.

O artigo está organizado como segue: a seção 2 apresenta o referencial teórico de avaliação e auto-regulação da aprendizagem; a seção 3 apresenta a ferramenta. Por fim, são apresentados os estudos de caso na seção 4 e na seção 5 são feitas algumas considerações finais.

\footnotetext{
${ }^{1}$ Disponível em http://www.moodle.org.br/

${ }^{2}$ Disponível em http://agora.tidia-ae.usp.br/portal
} 


\section{Auto-Regulação e Avaliação da Aprendizagem}

O insucesso escolar, caracterizado pelas reprovações, ocorre quando o estudante está abaixo dos padrões mínimos estabelecidos, indicando que o processo de ensinoaprendizagem necessita de regulação. Nesse sentido, a ação de regular pode ser definida como regularizar, estabelecer ordem ou ajustar [Ferreira, 2006]. A regulação da aprendizagem pode ser conduzida pelo professor ou pelo próprio estudante, sendo, nesse caso, denominada de Auto-Regulação. A auto-regulação da aprendizagem é conceituada, segundo Frison (2007) como o processo em que os sujeitos estabelecem metas que interagem com suas expectativas, desenvolvendo estratégias para alcançá-las, criando condições para que a aprendizagem se efetive.

Gonçalves (2010) defende que os alunos deverão ser explicitamente estimulados a deixar a atitude tradicional de espectadores da apresentação de conhecimentos e a se tornarem personagens principais na construção das suas próprias competências. Esta alteração torna necessário o conhecimento antecipado, por parte dos alunos, dos objetivos, ao nível das competências, do curso e/ou da unidade curricular que frequentam. Zimmerman (1989) afirma que para um aluno ser considerado auto-regulado este deve ser participante ativo metacognitivo, motivacional e comportamental em sua própria aprendizagem.

A auto-regulação pressupõe o uso de métricas que possam apoiar a identificação de ações regulatórias a partir das medidas obtidas, normalmente através da avaliação da aprendizagem. Segundo Pimentel (2006), o objetivo principal de uma avaliação deve ser diagnosticar as lacunas de aprendizagem do estudante a fim de se indicar o ponto de partida mais adequado para a instrução. O problema é que a principal medida de desempenho do estudante utilizada no sistema educacional, a média ou conceito final, esconde informações sobre o real desempenho do estudante no decorrer do processo avaliativo.

Esse trabalho propõe o uso de três métricas que serão detalhadas a seguir.

\section{NAC - Nível de Aquisição de Conhecimentos}

Pimentel (2006) define o NAC - Nível de Aquisição de Conhecimentos, como sendo uma medida que indica o grau de conhecimentos do aprendiz num determinado conteúdo, de um domínio de conhecimento, num dado instante. O NAC deve ser obtido a partir de diversas atividades de avaliação e é composto de duas partes: o índice de conhecimento e o índice de avaliação. Algumas propriedades definidas para o NAC são:

(a) Índice de conhecimento igual a zero indica ausência total de conhecimentos naquele conteúdo; (b) O índice de conhecimento pode crescer ou decrescer no decorrer do tempo, uma vez que a sua atualização se dará através de avaliações contínuas; (c) A fórmula básica para obtenção do índice de conhecimento do NAC pode ser média dos valores obtidos para o conceito em diversas avaliações ou pode-se utilizar médias ponderadas pelo tempo (avaliações mais recentes teriam mais peso).

\section{KMA - Knowledge Monitoring Accuracy}

O KMA - Knowledge Monitoring Accuracy (precisão no monitoramento do conhecimento) foi definido por Tobias e Everson, (2002) e mede a diferença entre a 
estimativa do aluno sobre seu conhecimento antes de realizar uma avaliação e seu nível de conhecimentos determinado pelo seu desempenho na avaliação. Segundo Gama (2004) o cálculo do índice KMA pode ser obtido através de variáveis como: quantidade de acertos na previsão do desempenho, quantidade de erros do tipo "grande otimista" (estima acertar e erra completamente a solução), quantidade de erros do tipo "grande pessimista" (estima errar e acerta completamente a solução), quantidade de erros do tipo "médio otimista" (estima acertar parcialmente e erra completamente ou se estima acertar completamente e acerta parcialmente a solução), quantidade de erros do tipo "médio pessimista" (estima acertar parcialmente e acerta completamente ou se estima errar e acerta parcialmente) e quantidade de problemas envolvidos na avaliação.

A Figura 1 mostra os significados dos valores de KMA. O valor do KMA está limitado no intervalo de -1.00 a 1.00 sendo que para valores compreendidos no intervalo de -0.25 a -1.00 o KMA é considerado Baixo, ou seja, o aprendiz não estima corretamente seu conhecimento na maioria das situações. Para valores compreendidos entre 0.50 e -0.25 , o KMA é Médio, ou seja, algumas vezes o aprendiz estima corretamente seu conhecimento, mas frequentemente comete erros médios ou grandes nas estimativas. E para valores compreendidos entre 1.00 e 0.50 o KMA é Alto significando que na maioria das vezes o aprendiz estima corretamente o seu conhecimento.

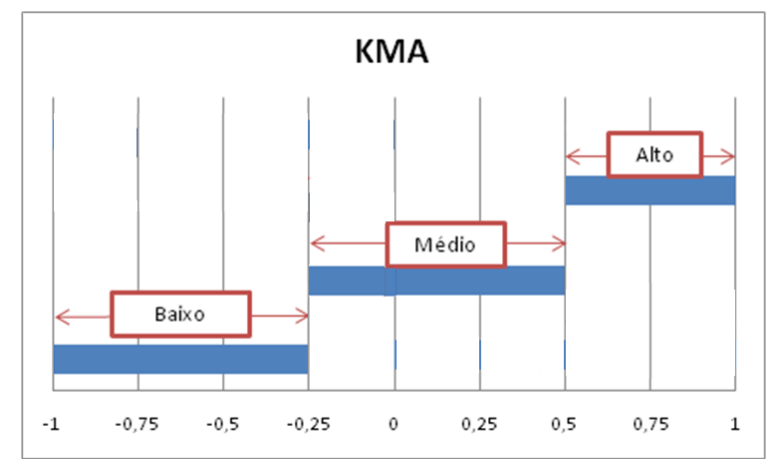

Figura 1 - Gráfico da Interpretação dos valores de KMA

\section{KMB - - Knowledge Monitoring Bias}

$\mathrm{O}$ KMB - Knowledge Monitoring Bias (viés no monitoramento do conhecimento) foi definido por Gama (2004) e mede e identifica o tipo de desvio do aprendiz, sendo possível saber se o aluno é pessimista, aleatório ou otimista. Este tipo de informação permite uma precisão mais realista no monitoramento da aprendizagem e possibilita ao professor dar feedback mais adequado para cada aluno. Para o cálculo do KMB utilizam-se as mesmas variáveis descritas para o KMA.

A Figura 2 mostra o significado dos valores de KMB. O cálculo do KMB está relacionado ao do KMA e a faixa de valores também varia entre 1.00 e -1.00 . No entanto, os valores do KMB demonstram um significado diferente do KMA. Para os valores compreendidos no intervalo de 0.25 a 1.00 o aprendiz é considerado Otimista, pois tende a estimar que pode resolver o problema mas não o faz na maioria das situações. Os valores compreendidos entre 0.25 e -0.25 o aprendiz estima seu conhecimento ora de maneira otimista, ora de maneira pessimista, sendo considerado 
Aleatório. O aluno será considerado Pessimista, ou seja, tende a estimar que não pode resolver o problema mas o faz com sucesso em muitas situações, quando o valor do $\mathrm{KMB}$ estiver compreendido entre -1.00 e -0.25 . O aluno é considerado Realista quando o KMA for alto.

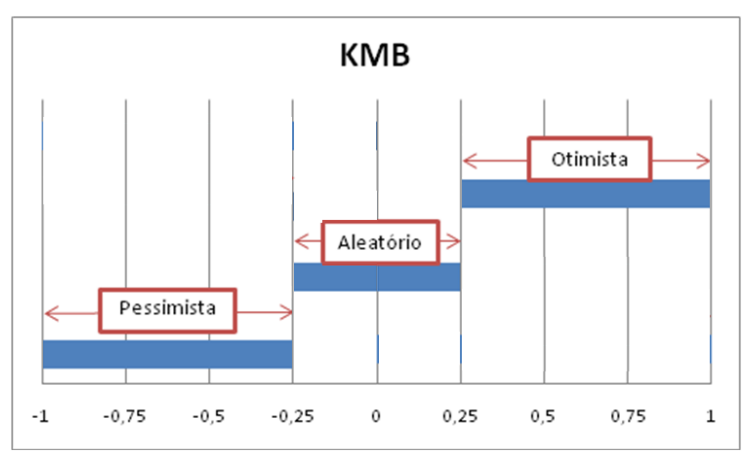

Figura 2 - Gráfico da Interpretação dos valores de KMB

\section{A Ferramenta SRS - Self Regulation System}

Essa seção apresenta a ferramenta SRS - Self-Regulation System (sistema para autoregulação da aprendizagem), que incorpora mecanismos para propiciar a auto-regulação da aprendizagem. A Figura 3 apresenta a arquitetura da ferramenta SRS composta por três módulos: Módulo Diagnóstico, Módulo Contínuo e Módulo de Acompanhamento. Estes módulos, em sua totalidade, têm as características e funcionalidades do modelo conceitual proposto por Pimentel (2006) e são descritas a seguir:

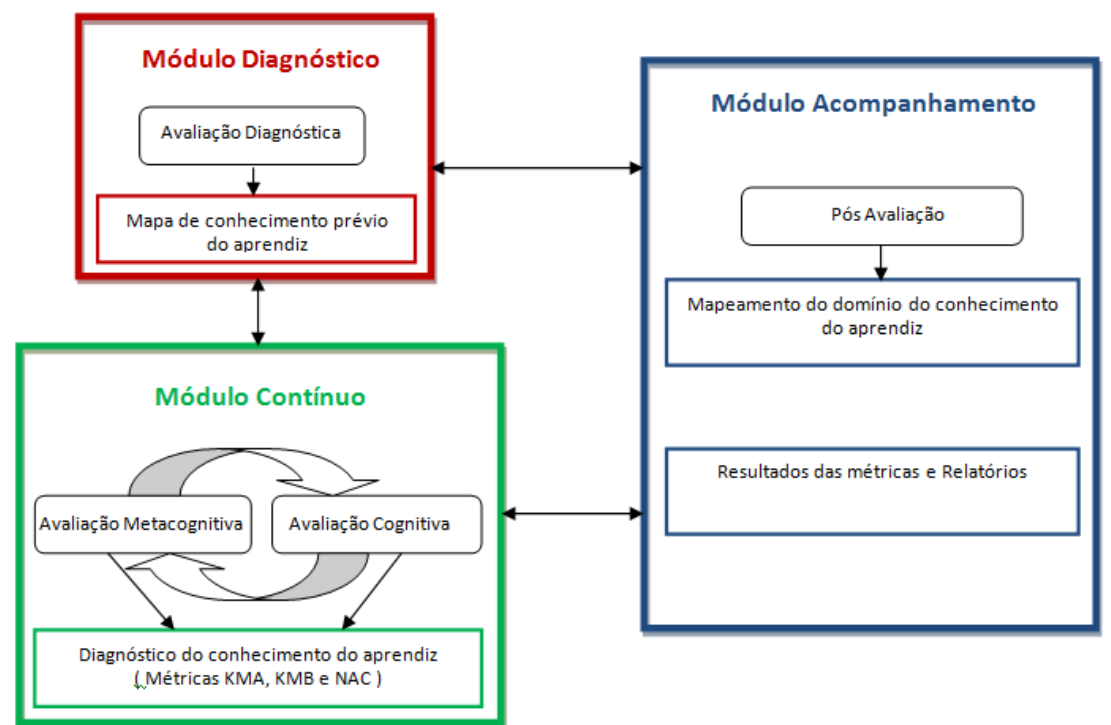

Figura 3 - Arquitetura Geral da Ferramenta de Auto-Regulação

\section{- Módulo Diagnóstico}

O submódulo Avaliação Diagnóstica do Módulo Diagnóstico, através de testes e/ou entrevistas, identifica o grau de confiança do aprendiz quanto aos conceitos envolvidos na disciplina na qual se encontra matriculado. Dessa forma, o sistema terá informações do conhecimento prévio do aprendiz que servirá de apoio para mapear o domínio do 
conhecimento do aprendiz e na interpretação dos resultados das avaliações metacognitivas.

\section{- Módulo Contínuo}

O Módulo Contínuo é similar ao Modelo de Avaliação de Pimentel (2006). Através das avaliações Cognitivas e Metacognitivas obtêm-se um diagnóstico do conhecimento do aprendiz na qual este será classificado com base nos valores das métricas KMA, KMB e NAC. Pode-se obter uma tabela com os dados das questões avaliadas e com base nas métricas classificar o aprendiz com a finalidade de orientá-lo e conscientizá-lo à autoregulação de sua aprendizagem. Contudo, as avaliações metacognitivas e cognitivas devem ser sequenciais e ordenadas para terem o efeito esperado e, assim, poder gerar resultados dos estudantes.

O módulo contínuo é iterativo, ou seja, cada ciclo de avaliações metacognitiva e cognitiva completa-se uma iteração. Desta forma, podem-se calcular as métricas KMA, KMB e NAC do aprendiz e assim obter seu diagnóstico de conhecimento. O professor pode submeter os aprendizes a quantas iterações julgar necessário, cobrindo todos os conceitos envolvidos no domínio de conhecimento em questão. Deseja-se, ao final de cada iteração, que o professor apresente os resultados aos alunos dando-lhes o feedback adequado. Também pode ser realizada, ao final da iteração, a reflexão pós-avaliativa compreendida pelo módulo de acompanhamento.

\section{- Módulo de Acompanhamento}

O Módulo de Acompanhamento agrega todas as informações dos módulos diagnóstico e contínuo e fornece o mapeamento do domínio do conhecimento do aprendiz. A reunião desses dados funciona como proposto no Modelo Acompanhamento do modelo de Pimentel (2006), mais precisamente o mecanismo de regulação. O módulo de acompanhamento contempla o submódulo Pós-Avaliação. Nessa etapa o aluno é submetido a um diálogo reflexivo na qual fornece informações sobre a utilização de todos os processos avaliativos contidos na ferramenta. $\mathrm{O}$ aprendiz é incitado a refletir sobre sua aprendizagem e como cada etapa do processo de auto-regulação o ajudou. Dessa forma, o educador possui informação para adequar seu processo de ensino a cada perfil de estudante.

Esses três módulos combinados e concluídos cumprem as etapas propostas no sistema de fases de Zimmerman (1989). O módulo diagnóstico coincide com a fase de conhecimentos prévios, o módulo contínuo com a fase de desempenho ou controle volitivo e o módulo de acompanhamento com a fase de auto-reflexão, como mostra a figura 4 .

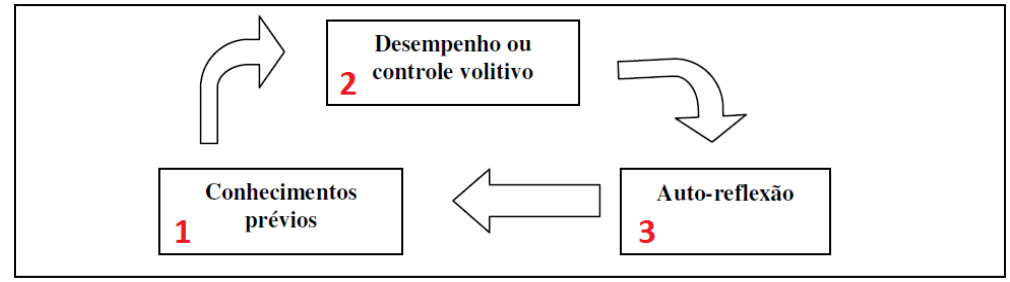

Figura 4 - Fases do sistema de auto-regulação da aprendizagem Adaptado de Zimmerman (1989) 
Para que esse ciclo seja utilizado e para garantir os resultados da aprendizagem do estudante por meio de suas métricas, é necessário seguir um fluxo ordenado de aplicação das avaliações. $\mathrm{O}$ estudante deve realizar a avaliação metacognitiva primeiro, na qual estima seu conhecimento, e logo em seguida realiza a avaliação cognitiva. $O$ confronto dos resultados das duas avaliações fornecem os valores das métricas KMA, KMB e NAC do estudante. A Figura 5 apresenta as avaliações disponíveis na área restrita do estudante.

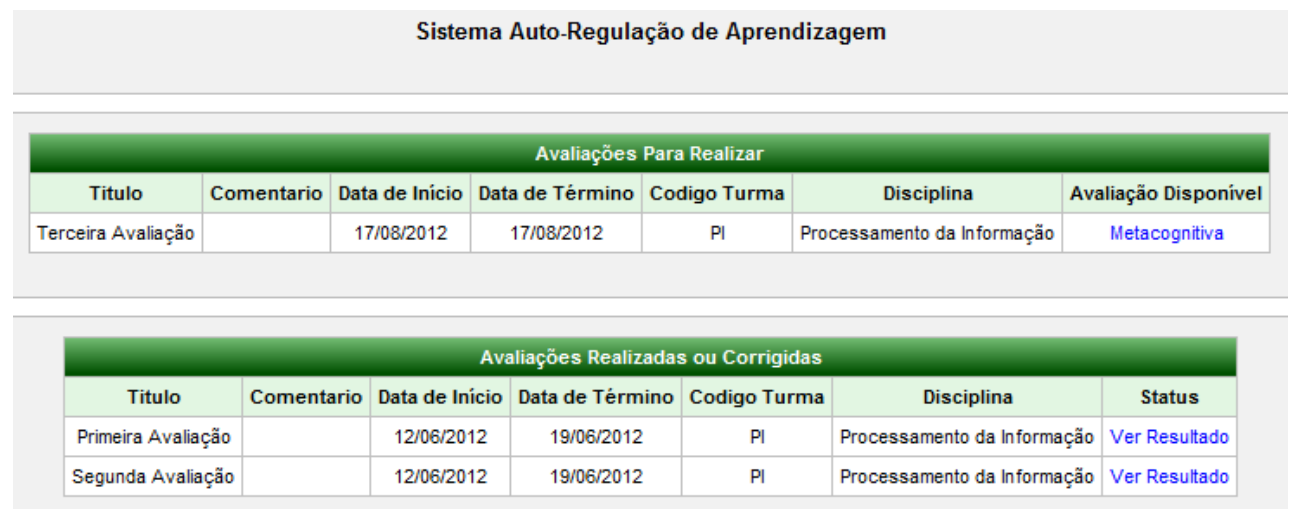

Figura 5 - Quadro de avaliações a serem realizadas pelo estudante

O professor poderá manipular as atividades e avaliações apenas através da dependência hierárquica dos conceitos de um domínio de conhecimento, ou seja, o educador deve, ao criar ou utilizar uma Unidade de Avaliação, associá-la aos conceitos na qual as compõem. Com isso, o aluno saberá a cada questão a ser resolvida o que exatamente está sendo avaliado, quais conceitos estão sendo exigidos, qual o grau de dificuldade e a profundidade do conhecimento esperado naquele tema de abordagem. Para isso, o ambiente deverá ser capaz de associar cada Unidade de Avaliação (UA) a seu conteúdo específico. Os ambientes virtuais de aprendizagem não fornecem um módulo de conteúdo que faça uma associação direta e objetiva dessas UA's. A ferramenta SRS tem um módulo na qual o professor associa a UA com seus respectivos conteúdos.

Com os resultados de todas as avaliações diagnósticas, cognitivas e metacognitivas e através das métricas utilizadas, o aluno terá condições de refletir sobre a capacidade de compreender seu conhecimento e saber resolver uma questão proposta. Os valores de desempenho obtidos pelas métricas KMA, KMB e NAC serão exibidos em forma de relatório e a cada iteração do estudante com os processos avaliativos esses valores são atualizados. Após cada iteração, ou seja, ao final de cada correção da avaliação cognitiva pelo professor são disponibilizados aos estudantes os resultados avaliativos dessa etapa. A Figura 6 mostra a tela dos resultados das métricas disponíveis aos aprendizes.

Após realizadas todas as avaliações (diagnósticas, metacognitivas e cognitivas) pelo aluno, uma Pós-Avaliação será realizada com o objetivo de fazer o aluno refletir sobre todas as métricas que lhe foi apresentado. Na interpretação dos resultados nem sempre fica claro ao estudante o que realmente significou para sua aprendizagem as 
métricas apresentadas. Portanto, um Diálogo Reflexivo com o Estudante será feito para observar se este tem ciência dos resultados avaliativos na regulação do seu aprendizado.

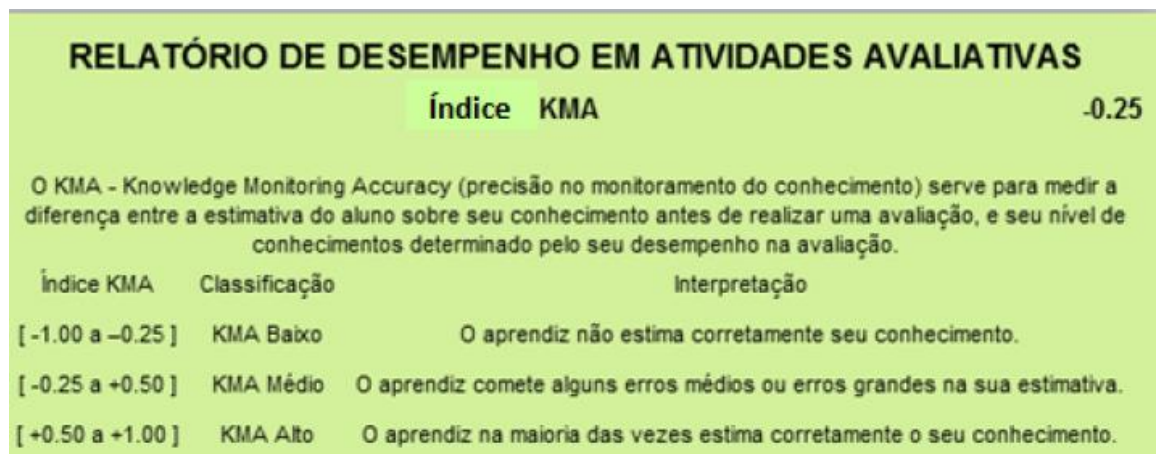

Figura 6 - Tela dos resultados avaliativos do aprendiz

As respostas destas questões ajudarão o estudante a refletir sobre seu processo de auto-regulação da aprendizagem. Além disso, fornecerá informação ao professor sobre feedback e sobre as interpretações dos resultados pelos alunos das métricas que obtiveram. Como resultado final o aluno terá a sua disposição um relatório contendo suas métricas, suas classificações, os conceitos corretos em cada avaliação e uma explicação do que é e como funcionam todas essas medidas. Com essas informações mais o feedback do professor, o estudante tem uma fotografia do conhecimento adquirido, chamado mapa. Esse mapa será construído a partir da união de todas as informações e métricas obtidas nas avaliações. Através do mapa o aluno terá como visualizar as deficiências e falhas da sua aprendizagem. A cada iteração o mapa é atualizado.

\section{Estudos de Casos}

Foram realizados dois estudos de casos em cenários distintos. O primeiro utilizou-se de ferramentas existentes no Tidia-Ae com o intuito de experimentar o uso das etapas avaliativas de uma forma integrada e das métricas metacognitivas e cognitivas no processo de auto-regulação da aprendizagem pelo estudante. $\mathrm{O}$ segundo foi realizado utilizando a ferramenta SRS a fim de experimentar e testar a sua dinâmica num cenário próximo do real.

Os estudos de casos seguiram uma sequência lógica de etapas. Chamou-se de etapa, nesse estudo de caso, cada submódulo dos módulos inicial, contínuo e conhecimento. Assim, as etapas dos estudos de casos foram:

- Etapa 1: Avaliação Diagnóstica (componente do módulo diagnóstico)

- Etapa 2: Avaliação Metacognitiva (componente do módulo contínuo)

- Etapa 3: Avaliação Cognitiva (componente do módulo contínuo)

- Etapa 4: Pós-Avaliação (componente do módulo de acompanhamento)

No primeiro estudo de caso, realizado no ambiente TIDIA-Ae, os alunos foram submetidos a três iterações; no segundo estudo de caso, realizado na ferramenta SRS, os alunos efetuaram duas iterações. Todas as etapas foram realizadas via online e os dados coletados armazenados em banco de dados. A etapa "avaliação diagnóstica" do módulo diagnóstico e a etapa "pós-avaliação" do módulo de acompanhamento podem ser 
aplicadas sempre que o educador achar conveniente. A Figura 7 representa o fluxo de aplicação das avaliações.

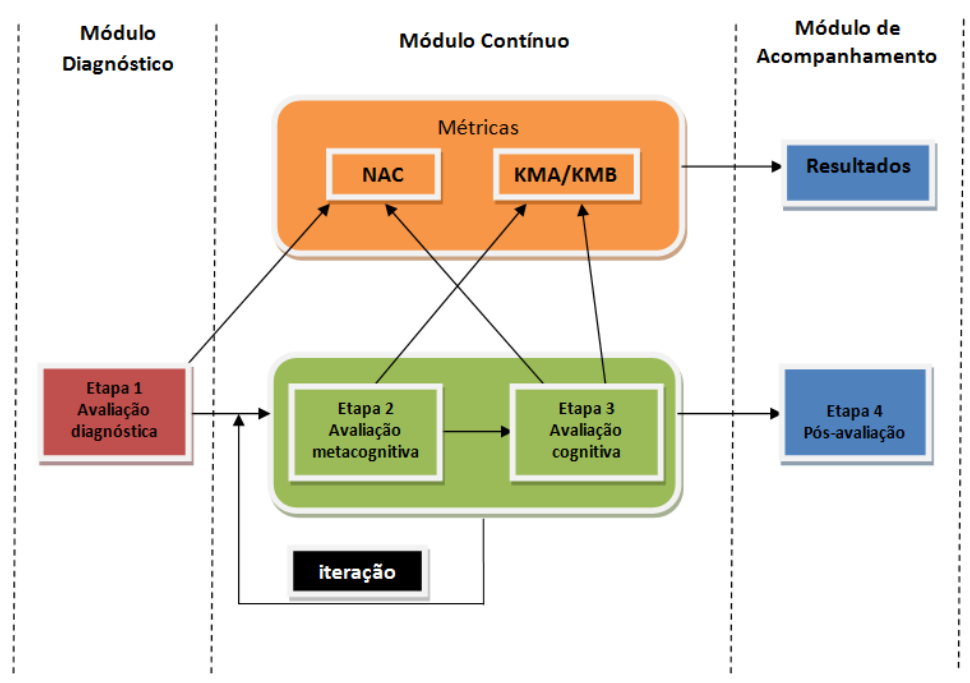

Figura 7 - Fluxo de aplicação das etapas avaliativas e geração das métricas

Ao final das quatro etapas realizadas em todas as iterações, o professor foi capaz de fazer uma previsão quanto a um possível desempenho dos alunos na avaliação somativa, realizada ao final do curso, interferindo, assim, na conduta das aulas. Os resultados dessa avaliação foram comparados aos resultados obtidos nas etapas avaliativas (avaliação formativa) realizadas pelos alunos e o diagnóstico foi conclusivo: os alunos cujos valores do KMA foi alto (aluno auto-regulado) e os alunos que apresentaram evolução positiva durante as iterações, obtiveram percentual de acerto superior a $70 \%$ na avaliação somativa. Isso mostrou uma interferência direta do modelo de avaliações integradas e das métricas geradas no aprendizado do estudante.

Além disso, os resultados desse questionário de pós-avaliação mostraram, segundo os alunos, que a ferramenta os ajudou a organizar melhor seus estudos. Além disso, a maioria dos alunos (cerca 80\%) entenderam os valores obtidos nas métricas e consideraram os feedbacks fundamentais para o processo de auto-regulação da aprendizagem.

Portanto, uma arquitetura pedagógica baseada na auto-regulação pode fornecer ao estudante um conjunto de informações capaz de envolvê-lo mais ativamente no processo de aprendizagem. Assim, ferramentas que propiciem um diálogo reflexivo com o estudante sobre o seu nível atual de aquisição de conhecimentos são necessárias. $\mathrm{Na}$ Educação a Distância isso tende a ser ainda mais relevante dada a ausência física do professor. Assim, um acompanhamento contínuo do processo, através de avaliações cognitivas e metacognitivas enriquecida por um diálogo reflexivo pode dar ao estudante uma sensação de maior presença do docente, tutores, etc.

\section{Considerações Finais}

Esse trabalho contribuiu com a implantação de uma ferramenta apoiada por tecnologia computacional e seguiu um fluxo lógico ordenado de realização das avaliações na qual possibilitou estudantes e professores acompanharem o nível de aquisição de 
conhecimento desses estudantes fornecendo subsídios para a consciência do processo de auto-regulação da aprendizagem. Os resultados desse acompanhamento permitem ao docente, durante o processo de ensino-aprendizagem, adotar medidas diferenciadas para ajudar os aprendizes a corrigir concepções errôneas. Além disso, a pós-avaliação permite uma reflexão do estudante quanto a sua aprendizagem, podendo levá-lo à autoregulação.

Como trabalhos futuros propõem-se a ampliação dos tipos de unidade de avaliação. A questão dissertativa é o único tipo de UA aceita pela ferramenta SRS. A geração de um banco de dados capaz de suportar outros tipos de unidades avaliativas como múltipla escolha, associação e falso e verdadeiro é um trabalho futuro desejável. Outro trabalho a ser realizado com a ferramenta SRS é sua integração a um AVA. O próximo desafio será acoplar essa ferramenta a um AVA como o Moodle ou o Tidia-Ae, por exemplo, aumentando, assim, os recursos desse ambiente de aprendizagem como a importação de unidades de avaliação.

\section{Referências Bibliográficas}

BRANSFORD, J. D.; et al. (2003) How people learn - brain, mind, experience and school. Washington: National Academy Press.

FERREIRA, A. B. H. (2006) Novo Dicionário da Língua Portuguesa. Nova Fronteira.

FLAVELL, J. H. (1979) Metacognition and cognitive monitoring: A new area of cognitivedevelopmental inquiry. American Psychologist, 34, 906-911.

FRISON, Lourdes M. B. (2007) Auto-Regulação da Aprendizagem. Ciência e Conhecimento revista eletrônica da Ulbra São Jerônimo - vol. 02, pedagogia, a.1

GAMA, C. (2004) Towards a model for Metacognition Instruction in Interactive Learning Environments. Tese (Doutorado). University of Sussex, Inglaterra.

GONÇALVES, I. C. (2010) Contributos dos Modelos da Auto-Regulação da Aprendizagem para a formação de Alunos e Professores no Ensino Superior. Disponível em https://dspace.ist.utl.pt/bitstream/2295/652736/1/ResapesComu nicacao.pdf

PIMENTEL, E. P. (2006). Um modelo para avaliação e acompanhamento contínuo do nível de aquisição de conhecimentos do aprendiz. Tese de Doutorado. Instituto Tecnológico de Aeronáutica. São José dos Campos: ITA.

TOBIAS, S.; EVERSON, H. T. (2002). Knowing what you know and what you don't: further research on metacognitive knowledge monitoring. College Board Research Report 2002-3, College Entrance Examination Board: New York.

ZAIANE, O. R.; LUO, J. (2001) Towards evaluating learners' behaviour in a web-based distance learning environment. Advanced Learning Technologies. Proceedings. IEEE International Conference.

ZIMMERMAN, B. J. (1989) A social cognitive view of self-regulated learning. $J$. Educ.Psychol. n. 81. 